\title{
Should our practice participate in the New CMS Primary Care First Initiative?
}

\author{
Mikila Lawless* \\ Harden Communications Partners, 520 3rd St. Ste. 204, Oakland, CA 94607, USA
}

The recently announced CMS Primary Care First (PCF) initiative continues to build on the incremental shift of care delivery from volume to value through improving outcomes, reducing utilization and controlling costs. By incentivizing primary care practitioners through a combination of performance-based payments and a flat primary care visit fee, CMS expects this new alternative payment model will help reduce costs while minimizing the administrative burden associated with revenue cycle functions for practices. Practice and payer applications for PCF are slated to begin in 2019, and PCF starts in January 2020.

As primary care practitioners consider participating in PCF, it will be prudent to determine their practice's tolerance to assume the financial risk for population management, as well as broadly assess their readiness to deliver comprehensive primary care to achieve PCF's targets. Deciding on the value of PCF versus other payment models involves multiple factors, such as having a strong foundation for data analytics and sufficient infrastructure for enhanced health care services.

\section{PCF: A Primer}

PCF is a voluntary, five-year, performance-based payment model that rewards primary care practitioners for improving quality and access and reducing costs. It is rooted in the core tenets of CMS's Comprehensive Primary Care functions: access and continuity; care management; comprehensiveness and coordination; patient and caregiver engagement; and planned care and population health.

PCF participants will find it is based on CMS's existing Comprehensive Primary Care Plus (CPC+) model, launched in 2017, which emphasizes doctor-patient relationships, enhanced care for high-need and chronically ill patients and rewards for improved health outcomes. The key difference with PCF, as its name implies, is that it focuses on primary care and serious illness services.

Under PCF, practices will be evaluated against a set of clinical quality and patient experience measures to receive payments based on two models:

PCF Payment Model: Targeted at small- to medium-sized practices, this payment model consists of a population-based payment, flat primary care visit fee, and a performance-based adjustment.

High Need Populations Payment Model: This model provides higher payments to practices that specialize in care for high-need, seriously ill populations who lack a primary care practitioner and/or effective care coordination. This includes services such as acute inhome care services and palliative care.

For practices, initial eligibility for PCF will be limited to 26 regions, including 18 existing $\mathrm{CPC}+$ regions plus eight states: Alaska,
California, Delaware, Florida, Maine, Massachusetts, New Hampshire and Virginia. Payers may participate in all 26 initial PCF regions.

\section{Is Your Practice Ready for PCF?}

Evaluating a practice's current infrastructure from a people, process, and systems framework will help determine whether it can sufficiently meet the targets of PFC's value-based strategies. The following are the recommended inputs and capabilities needed to maximize the benefits of participating in PCF.

- Processes: The foundation of a value-based program rests on an effectively designed care management model predicated on population size and corresponding risk. A risk-stratified care management program requires efficient workflows delivering clinically appropriate care to the selected patient population. Workflows reflecting team-based care coordination are essential to integrate behavioral health care and assess and support patient's psychosocial needs -- both of which are fundamental to PCF.

- People: Care coordination requires transitioning to a team-based care model in which all clinical disciplines and non-clinical staff function as a single, coordinated unit to deliver patient-centered care. As part of the primary care redesign, implementing a regular process for patients and caregivers to advise practice improvement is part of PCF. These types of transformational organizational changes, with feedback loops for continuous improvement, require strong leadership and sponsorship from key stakeholders. It is vital to engage physician champions to spearhead these cultural changes in the organization alongside their operations counterparts.

- Technology: The role of technology and data is central to valuebased programs. After the spate of EHR adoptions of the past decade, organizations have quickly realized that technology strategies for effective population management extends well beyond EHR. Robust data analytics and reporting capabilities are a necessity for both cost and quality data. From a patient engagement standpoint, PCF calls for 24/7 access to a care team practitioner with real-time access to the EHR to meet access and continuity functions of primary care. Practices will need to continue making technology investments in patient communication capabilities in areas such as telemedicine that facilitate early intervention and

${ }^{*}$ Correspondence to: Mikila Lawless, Harden Communications Partners, 520 3rd St. Ste. 204, Oakland, CA 94607, USA, Tel: 510-488-6663; 559-708-3664; E-mail: mlawless@hardenpartners.com

Received: August 06, 2019; Accepted: September 05, 2019; Published: September 09, 2019 
proactive management, particularly among patients with chronic conditions.

As the push for greater accountability in care delivery continues, primary care practitioners should review their capacity to deliver on their PCF goals and understand the investments required in terms of processes, people and technology. To learn more about PCF, including CMS plans and timing for practice and payer applications, click the CMS fact sheet here.

Copyright: @2019 Lawless M. This is an open-access article distributed under the terms of the Creative Commons Attribution License, which permits unrestricted use, distribution, and reproduction in any medium, provided the original author and source are credited. 